\title{
LOS APORTES DE LA FENOMENOLOGÍA AL EJERCICIO PSICOTERAPÉUTICO. UNA REVISIÓN HISTÓRICA
}

\author{
THE CONTRIBUTIONS OF PHENOMENOLOGY TO THE \\ PSYCHOTHERAPEUTIC EXERCISE. A HISTORICAL REVIEW \\ Cristóbal Andrés Pacheco Pacheco \\ Universidad Autonoma de Chile. Chile
}

Cómo referenciar este artículo/How to reference this article:

Pacheco Pacheco. C. A. (2019). Los aportes de la fenomenología al ejercicio psicoterapéutico. Una revisión histórica. Revista de Psicoterapia, 30(112), 147-164. https://doi.org/10.33898/rdp.v30i112.254

\begin{abstract}
Resumen
El desarrollo de una construcción histórica, basada en el rescate de los aportes que surgen desde la tradición de la fenomenología, resulta un ejercicio necesario para la comprensión de una posibilidad de abordaje enfocada en el rescate de la vivencia subjetiva y particular. Desde las bases epistemológicas de la fenomenología, es posible obtener una definición ontológica que incorpora elementos de carácter ideográfico a la comprensión del individuo, comprometiendo una inmersión en un ejercicio centrado en las posibilidades comprensivas que brinda un encuentro con lo fáctico de la experiencia personal. El presente trabajo compone un registro histórico de los alcances psicoterapéuticos de exponentes tales como Karl Jaspers, Viktor Frankl, Alfried Längle y Giampiero Arciero, constituyendo una revisión que plasma en detalle sus aportes particulares a la intervención psicológica, mediante la inmersión en la vivencia particular.

Palabras clave: fenomenología, vivencia particular, registro histórico, posibilidades comprensivas.
\end{abstract}

\begin{abstract}
The development of a historical construction, based on the rescue of the contributions that arise from the tradition of phenomenology, is a necessary exercise for the understanding of a possibility of approach focused on the rescue of the subjective and particular experience. From the epistemological basis of phenomenology, its possible to obtain an ontological definition that incorporates elements of a ideographic character to the comprehension of the individual, committing an immersion in an exercise focused on the comprehensive possibilities that provides a meeting with the factic of personal experience. This paper makes a historical record of the psychotherapeutic reaches of exponents such as Karl Jaspers, Viktor Frankl, Alfried Längle and Giampiero Arciero, constituting a review that reflects in detail their particular contributions to the psychological intervention, by immersion in the particular experience.

Key words: phenomenology, particular experience, historical record, comprehensive possibilities.
\end{abstract}




\section{Introducción}

En el contexto de la continua dominancia del paradigma de las ciencias naturales en psicoterapia, una alternativa se puede referir a la psicoterapia como una ciencia humana y la fenomenología, puede servir como una alternativa de abordaje a nivel clínico (Mook, 2010). La fenomenología, ofrece una perspectiva de lo que significa ser una persona en un mundo impregnado de lenguaje, historia y tradición, compartiendo dominios de interés desde una mirada psicoterapéutica y filosófica, en conceptos tales como juego, narrativa, comprensión e interpretación (Mook, 2010).

Desde una corriente psicopatológica, es importante destacar los aportes de Karl Jaspers, quien creo un campo de psiquiatría comprensiva, introduciendo la fenomenología a la práctica psiquiátrica, asociando el ejercicio terapéutico a un acercamiento desprejuiciado a un fenómeno mental, recomendando una "tranquila inmersión en los hechos de la vida psíquica, sin adelantarse a conclusiones" (Jaspers, 1965, p. 331). Jaspers plantea que, con el método explicativo, contamos con un acercamiento a la realidad clínica, como un físico estudia la materia o bien como quien estudia el tamaño de los ventrículos cerebrales o la medición del intelecto cuantitativamente (Jaspers, 1959). Por otra parte, con el método de la comprensión se cuenta con un acceso al fenómeno, que resiste nuestro entusiasmo de cuantificarlo, así como sentimientos o emociones personales y técnicas en general, permitiendo adentrarnos en el mundo del significado por sobre una comprensión lineal (Jaspers, 1959).

Se puede referir que los postulados establecidos por Heidegger (1997) son relevantes en el encuentro con la naturaleza propia del ser, brindando una importancia a la observación del hombre a partir de su historia y su vida fáctica, favoreciendo de esta manera un acercamiento a su desarrollo biográfico, nutrido del mundo de la vivencia misma en el contexto de la relación hombre-mundo.

En términos prácticos es menester visualizar el desarrollo de postulados ontológicos que permitan profundizar la comprensión de un ser inmerso en una existencia que va más allá de la propia conciencia, planteando la importancia del solo hecho de existir como determinante en la constitución individual. En términos prácticos, la búsqueda de la interpretación de las vivencias particulares, a partir de la comprensión de la experiencia subjetiva, entregara los primeros indicios de una propuesta terapéutica, fundada en la inmersión biográfica, más allá de la descripción sintomatológica.

Es ante lo anteriormente referido, que se ha articulado una revisión de los planteamientos teóricos de exponentes en fenomenología clínica tales como Karl Jaspers, Viktor Frankl, Alfried Längle y Giampiero Arciero, propiciando un acercamiento a sus planteamientos que configuran modelos de acercamiento a la experiencia biográfica a nivel clínico. Se ha mantenido un orden histórico que permita al lector, interiorizarse respecto a aspectos de carácter biográfico, teóricos y técnicos de los autores comprometidos en la presente publicación. 
La Comprensión como Cuestionamiento del Fenómeno del Diagnóstico: el Método Biográfico y su Encuentro con la Realidad Subjetiva

Karl Jaspers presenta una importancia significativa para el desarrollo de la psiquiatría a nivel histórico, con el desarrollo de su libro Psicopatología General (1913), demarca la totalidad de los campos de la psicopatología a nivel general, como también los hechos y los puntos de vista de esta ciencia (Jaspers, 1959). En el desarrollo de una propuesta psicopatológica estructurada, establece una diferenciación de la tradicional psiquiatría francesa, estableciendo una metodología de carácter reflexivo que evidencia en gran parte su amistad y planteamientos en común con filósofos de la talla de Martin Heidegger (Chaslin, 1912).

El acercamiento de Jaspers a la fenomenología ocurre en 1909, con su lectura de las investigaciones lógicas de Edmund Husserl, libro que brindaría una fuerte influencia para el desarrollo de un proceso reflexivo, en el cual logra visualizar elementos clave en la formación de sus propuestas a nivel psicopatológico, visualizando la posibilidad mediante la fenomenología de describir la vivencia de enfermos mentales, indicando que el hecho de volver a las cosas mismas a partir de este método, como también abstraerse de prejuicios y esquematismos le resultaba liberador (Jaspers, 1951). Es importante destacar que si bien durante el desarrollo de sus postulados, Jaspers presenta un acercamiento a los planteamientos establecidos por Husserl, posteriormente, cuenta con un mayor acercamiento a lo propuesto por Heidegger (1997), en vista de que no realiza las reducciones propuestas por el autor, como tampoco contaba con el afán de desarrollar una nueva fundamentación radical de todas las ciencias, como pretendía Husserl a partir de la fenomenología (Husserl, 1962).

Como puntos representativos de la fenomenología de Jaspers pueden describirse los siguientes:

1. Hacer presente (Vergegenwärtigung) aquello que sucede al interior del enfermo, lo que auténticamente vivencia como se da en su conciencia (Jaspers, 1912).

2. La postura fenomenológica, elimina toda teoría, construcción psicológica o teoría en torno a presuntos procesos cerebrales, siendo tal ausencia de prejuicio producto de un gran esfuerzo por parte del tratante (Jaspers, 1912).

3. La fenomenología se centra en el empatizar (Einfühlen), co-vivenciar, adentrarse afectivamente o transponerse (Hineinversetzen) (Jaspers, 1959).

4. Primacía de la descripción aséptica, buscando profundizar al interior de los fenómenos vividos, de esta manera surgen estructuras de la vida que son pre-objetivos o pre-temáticas (Blankenburg, 1984).

5. Referencia a la totalidad interna de la experiencia psicológica.

6. En base al acercamiento a Heidegger, surge la necesidad del desarrollo de la comprensión, la cual busca establecer una diferencia de la ciencia objetivista e inductiva, basando el desarrollo de un método comprensivo 
como método de acercamiento al conocimiento de la realidad individual (Jaspers, 1959).

Es justo denominar el desarrollo de un método descriptivo a nivel clínico por parte de Jaspers, método que aspira a desarrollar una aproximación a la experiencia de la vida a partir de la comprensión, como también la interpretación desprejuiciada de la vida del paciente desde su dimensión subjetiva. En el desarrollo del presente método, es menester la ausencia inicial de explicaciones por parte del especialista de acuerdo a lo establecido por Jaspers, quien debe enfocarse en una tarea de observación y descripción de los fenómenos relatados por el paciente, sin embargo ello no responde a la idea de prescindir de un acercamiento ateórico a la vivencia particular, siendo necesaria la utilización de una conocimientos asociados a psicopatología "normal y anormal" por parte del especialista (Parnas, Sass y Zahavi, 2013).

Es importante referir como antecedente significativo en la obra del presente autor, la elaborada critica desarrollada en su conferencia "Sobre la crítica del Psicoanálisis" (1950), en ella establece una fundada observación, basada en la utilización de la palabra teoría, lo cual disgustaba profundamente a Jaspers, indicando que la psicología en la manera que se configura en su saber sobre el alma, no es una ciencia pues no puede experimentar en un sentido pleno (Hersch, 2002). La observación en cuanto a la causalidad de enfermedad, se funda en que la causalidad es técnica y constituye un problema de tratamiento psicoterapéutico más o menos eficaz, por otra parte, el sentido es algo existencial que no acepta traducción ni adaptación a fórmulas posibles, siendo la personalidad moral tanto del enfermo como la del tratante la que se pone en juego en la enfermedad mental. (Hersch, 2002).

Dentro de las criticas establecidas por Jaspers respecto al Psicoanálisis, se encuentran:

1. Todo lo que sucede al hombre tiene un sentido, sin embargo, traducir el sentido a síntoma, absolutizar el significado de los síntomas e interpretar una biografía entera por la ausencia o presencia de algunos de ellos, es degradar al individuo (Jaspers, 1950).

2. Existe un totalitarismo Psicoanalítico, el cual proviene de no separar cuerpo y alma, pretendiendo el psicoanálisis un conocimiento total del ser humano, el cual acaba en totalitario (Jaspers, 1950).

3. El Psicoanálisis presupone que existe un estado humanamente perfecto, denominado salud, lo cual es cuanto menos dudoso, considerando el valor dinámico asociado al concepto de salud (Jaspers, 1950).

En síntesis, la perspectiva elaborada por Jaspers en cuanto a la comprensión y abordaje a nivel psicopatológico, ha contado con un valor inconmensurable en la historia de la salud mental, favoreciendo la articulación de una comprensión biográfica del individuo, enfocada en la importancia de favorecer una comprensión descriptiva de los fenómenos vivenciados por el sufriente. Esta aproximación 
responde al desarrollo de una visión ontológica, basada de manera directa en los supuestos establecidos por Husserl y Heidegger, sin embargo, al ser utilizados en un contexto clínico, plantean un novedoso giro al quehacer profesional, basado regularmente en paradigmas técnico-explicativos.

Si bien se puede indicar que la crítica formulada al psicoanálisis por parte de Jaspers, puede resultar en la actualidad algo "excesiva" o bien "invalidante" hacía las teorías formuladas, es relevante aseverar que el propósito de dichas declaraciones, se basan en una posibilidad de regresar la intervención al ser humano, como sujeto activo y protagonista de una vivencia que debe primar por sobre una teorización determinista e inductiva en cuanto al fenómeno de la psicopatología.

\section{La Búsqueda de Sentido como Tarea Fundamental en el Desarrollo Vital}

Del peculiar y a veces paradójico fenómeno del desarrollo humano, se desprenden conceptos, eventos, momentos, ideales y por qué no decirlo "misiones", proyectos que trazan horizontes personales, que impactan la vida de quienes demarcan un ideal, cuyo destino inherente en la búsqueda del sentido de satisfacción personal. La voluntad de sentido corresponde una motivación primaria del ser humano, motivación que impregna de significados el acto de vivir, brinda fuerzas, define caminos y brinda razones para apoyar la propia existencia, es desde la presente perspectiva que Viktor Frankl (1926) desarrolla su método terapéutico, el cual aborda la búsqueda del sentido individual desde el ejercicio del análisis existencial.

En un inicio Frankl se ve atraído por los postulados establecidos por el Psicoanálisis de Sigmund Freud y la psicología individual de Alfred Adler, desarrollando lo que posteriormente sería la Logoterapia o también conocida como la tercera fuerza psicoterapéutica Vienesa. La Logoterapia puede ser comprendida como una propuesta centrada en hacer consciente la propia libertad del individuo, aumentando la responsabilidad ante la vida y asumiendo la angustia que es inherente al hecho de ser libre para dar respuestas (Salomón y Díaz del Castillo, 2015). Esta responsabilidad se asocia al hallazgo y vivencia de valores, de estar abierto al mundo de forma autentica, encontrándole un sentido a la vida (Salomón y Díaz del Castillo, 2015).

Es menester identificar la motivación principal de Viktor Frankl, asociada a la generación de la Logoterapia, la cual se asocia a una historia de vida donde el sentido de vida, presenta una orientación comprensiva a nivel biográfico, en palabras de Fizzotti (1977), se puede indicar: "Conocer la Logoterapia, nos obliga necesariamente a encontrarnos con su fundador, que es el primero que ha experimentado un su carne el tormento y la angustia de una vida privada de sentido, reuniendo los episodios que componen esta vida fascinante y misteriosa" (p. 16). Un episodio clave en la vida de Frankl, se asocia a su deportación a campos de concentración de Nazi, desde 1942 a 1945, espacio en el cual se ve fuertemente impregnado por un sufrimiento astronómico en proporciones, momento de su vida en el cual desarrolla 
la humanitaria tarea de prevenir suicidios y desarrollar procesos de contención con los prisioneros. Es a partir de esta vivencia que se desarrollan y constituyen los planteamientos del método propuestos por el autor, siendo "el sentido", la fuerza que contribuye a su supervivencia y posterior liberación en 1945, momento en el cual y a su regreso a Viena, escribe el Hombre en busca del sentido, obra magna que expone sus experiencias personales en los campos de concentración como también las bases de su modelo terapéutico y visión ontológica.

Sin embargo, también surge la necesidad de que el interés por la motivación y el sentido humano, no comienzan para Frankl en 1942, anteriormente y motivado por la influencia de Allers y Schwars, miembros de la asociación Adleriana, desarrolla un ensayo en el cual sintetiza sus principales ideas (Fizzotti, 1977).

1. Desarrolla una crítica filosófica al psicoanálisis de Freud, indicando que este solo atiende a las pulsiones del hombre, olvidando la totalidad corporal, psíquica y espiritual de la persona.

2. Establece que la actitud del terapeuta ante un determinado sistema filosófico, debe responder con argumentos filosóficos a las cuestiones de índole que le planteen sus pacientes.

3. Señala la existencia entre el tratamiento psicoterapéutico y los valores, indicando que la entrevista clínica no debe desatender este tema, refiriendo que no se trata de influir en la concepción filosófica de los pacientes sino de que ellos establezcan su propia jerarquía de valores para construir sobre ella su existencia futura.

4. El paciente ha de ser invitado a recurrir a todas sus posibilidades de libertad y responsabilidad.

Para acercarse a una definición más exacta de la práctica y orígenes de la Logoterapia, es importante mencionar que el vocablo griego logos, se define como: "razón intima de una cosa, fundamento, motivo", a nivel psicoterapéutico, el logos presentaría un papel más amplio, asociado a la posibilidad mediante la Logoterapia, de ampliar el campo visual del paciente para que descubra la gama de significados y de valores que está llamado a realizar, siendo el logos, el mundo espiritual, objetivo del sentido y los valores (Frankl, 1988). Desde esta visión, se puede comprender al mundo como un espacio ordenado, un cosmos, el cual viene a ser el correlato de la existencia personal, en cuanto espiritual y subjetivo (Frankl, 1986).

Se puede afirmar que Frankl en su insatisfacción por un planteamiento mecanicista-causal de la psicoterapia, asociado a la necesidad de considerar al hombre en un sentido global en sus dimensiones físicas, psíquicas y espirituales, fueron influyendo en la idea de definir un nuevo modelo de intervención psicoterapéutica (Bazzi y Fizzotti, 1989). Para la Logoterapía la motivación principal para el hombre es la búsqueda de un sentido a su propia vida, cuando se produce un vacío existencial como perdida de sentido, se puede producir un tipo de 
Neurosis que Frankl denomina como noógena (Frankl, 1994). La labor del terapeuta es contribuir a restablecer o encontrar su sentido de vida perdido, ya que en palabras de Frankl "Si lo quiere o no, si lo sabe o no, el hombre cree en un sentido mientras respira, hasta el suicida crea un sentido, aunque no sea en el de la vida o del seguir viviendo, es al menos en el del morir" (Frankl, 1994, p.115).

La Logoterapia se vuelve una terapia fenomenológica, partiendo desde un dialogo en un sentido socrático, que parte de una actitud de ignorancia y respeto por la figura consultante, propiciando el desarrollo de una interacción que permita el alcance de sus propias verdades y sentidos (Salomón y Díaz del Castillo, 2015). La mantención de una actitud fenomenológica, basada en el desarrollo de un dialogo desprejuiciado, propicia la anulación del uso de una técnica mecanizada, la cual responda a la implementación de una serie de estrategias o métodos para la obtención de resultados, más bien se asocia a una práctica vivencial que busca una relación directa y sincera con otro, en palabras de Viktor Frankl: "En el ámbito de la psicoterapia, lo que menos importa es el método o la técnica empleados en cada caso, el factor decisivo es más bien la relación entre médico y enfermo" (Frankl, 2003, p.51).

Para el desarrollo del método fenomenológico en la Logoterapia, se pueden describir de manera resumida los siguientes conceptos:

1. Epoche: El poner entre paréntesis toda creencia, juicio o idea particular, implica el ejercicio de autodistanciarse a modo de lograr reconocer el conjunto de creencias a priori, facilitando de esta forma el desarrollo de una suspensión. Esta suspensión no implica anular todo conocimiento o creencia, sino que conlleva distinguir que contenidos personales pueden entorpecer el desarrollo de una interpretación e intervención con la figura del consultante, esto es llamado diferenciación (Martínez, 2012).

2. Descripción: Se procede una vez realizado el ejercicio de suspensión y diferenciación a describir la experiencia del consultante, manteniendo una actitud fenomenológica, basada en una disposición afectiva (Gesinnung) (Scheler, 1957). La intención se asocia a percibir la vivencia descrita por otro en las esferas verbales, corporales y psíquicas, presentando una actitud apertura, manteniendo la presencia que garantice estar atento a otro espiritual (Frankl, 2007).

3. Horizontalización: El encuentro con relatos personales, se asocia a considerar que ningún aspecto de la descripción es más valioso que otro y en el desarrollo de la Logoterapia se busca descubrir y jerarquizas los significados particulares del consultante, siendo el quien defina su propósito y sentido a nivel personal.

4. Pregunta: Es mediante el desarrollo de la pregunta, que el dialogo socrático se fundamenta, además de representar el principal punto de encuentro con la vivencia particular, desde una perspectiva desprejuiciada, orientado siempre una intencionalidad orientada al saber y comprender 
(Salomón y Díaz del Castillo, 2015).

En síntesis, se puede afirmar que la búsqueda del sentido como motivación trascendental del ser humano, representa una búsqueda por la libertad y autonomía personal, constituyendo el sentido una fuerza movilizadora que brinda orientación y disposición al hecho de una continuidad existencial. Es propicio señalar que el acercamiento analítico existencial realizado por la Logoterapia, se ajusta a un encuentro con una dimensión de sentidos particulares, siendo desde un sentido fenomenológico, una oportunidad de reconocimiento del acto subjetivo de la vivencia personal, asociada a una motivación intrínseca que entrega significados y posibilidades individuales de desarrollo.

Es factible referir que la búsqueda del sentido a nivel individual, es solo una dimensión del desarrollo humano, si bien abarca una motivación fundamental, no incorpora toda la esfera de la composición ontológica, la cual se asocia necesariamente a múltiples factores y elementos más allá de la sola búsqueda de sentido, pudiendo describir la motivación o motivaciones humanas, como elementos propios de significados particulares, estos significados y necesidades pueden ser abordados en el encuentro dialógico establecido en una interacción de índole fenomenológica hermenéutica.

\section{EI Análisis Existencial y las Motivaciones Fundamentales de la Existencia}

La construcción de una visión ontológica, conlleva la oportunidad de visualizar el desarrollo humano desde variables tales como cultura, contexto histórico, influencia social y vivencias individuales, ocupando un principal lugar la concepción existencial, la cual agrupa una definición humana a partir de todos los determinantes antes mencionados, como también de la percepción particular de la vivencia encarnada. Desde una perspectiva práctica, el Análisis Existencial es una psicoterapia de tipo fenomenológica, enfocada en el respeto a la libertad de cada persona, generando nuevas oportunidades de enfrentamiento a la vida, a partir de la construcción de nuevos significados particulares.

Es en base a lo anteriormente referido, respecto a los planteamientos realizados por Viktor Frankl, que pueden distinguirse de manera clara los orígenes del Análisis Existencial, psicoterapia que responde una necesidad de desarrollar un trabajo enfocado en el establecimiento de un dialogo enfocado en la vivencia, no tanto en el recuerdo o pensamiento del paciente, para centrarse luego en la actualización emocional y posteriormente en la toma de decisiones y actitudes por parte de la persona y finalmente se pone énfasis en la actualización del actuar, como resultado de la toma de decisiones individuales (Längle, 1990).

Desde una dimensión histórico-teórica, Alfried Längle comienza siendo el principal discípulo de Viktor Frankl, en cuanto al desarrollo de una terapia basada en el Análisis Existencial, fundando la Sociedad de Logoterapia y Análisis Existencial, sin embargo, a comienzos de los años 90, decide renunciar a la presente sociedad, al considerar que la dirección de esta se estaba alejando de algunos postulados tanto 
a nivel clínico como en la formación de psicoterapeutas. (Längle y Traverso, 2013). Längle planteó que una teoría enfocada solo en el sentido, entregaba una base insuficiente para abordar todas las problemáticas a nivel psicoterapéutico, indicando que no todos los sufrimientos personales, se asocian a la perdida de sentido, es ante lo cual que desarrolla una teoría que plantea que existen cuatro motivaciones fundamentales que sostienen la existencia humana (Längle y Traverso, 2013).

La primera de estas motivaciones fundamentales, se asocia a la motivación de EI Ser, comprendiendo el hecho de ser, como una persona que vive, que se pregunta respecto a su propia existencia, como alguien que es dador y que recibe, constituyendo el hecho de la mera existencia (Längle, 2000). De la siguiente existencia surge la pregunta "Yo soy, ¿pero puedo ser?", las condiciones para la mantención o superación de esta motivación se asocian a la protección, la existencia de espacio para mi persona y la entrega de sostén que brinda confianza (Längle y Traverso, 2013). La ausencia de las condiciones antes mencionadas, producen reacciones psicodinámicas "coping", las cuales surgen como respuesta adaptativa y de supervivencia ante las demandas de un entorno que no satisface las condiciones asociadas a la presente motivación, la primera reacción de "coping" se asocia a una reacción básica evidenciada en la acción de Huida; la segunda reacción de tipo paradojal, se asocia al Ataque; la tercera reacción de tipo agresiva, se asocia al Odio y finalmente la última reacción vinculada a un reflejo de muerte, corresponde a la Parálisis (Längle, 2000).

Es a partir de la no satisfacción de estas necesidades es que pueden surgir a nivel psicopatológico, trastorno de ansiedad, traducidos en cuadros de Angustia, Fobia, Trastornos obsesivo compulsivos y Trastornos de Pánico (Längle, 2000).

Respecto a la segunda motivación fundamental, esta se asocia al acto de vivir, del libre fluir de la vida, la apertura al placer y disfrute, de esta motivación surge el siguiente planteamiento "Yo vivo, ¿pero me gusta vivir?", dentro de las condiciones para lograr una satisfacción y un disfrute de la vida se encuentran la cercanía física, la relación y el tiempo como condición necesaria para todo movimiento (Längle y Traverso, 2013). En el establecimiento de una relación con alguien o algo, se aprende y logra valorar la vida, se logra sentir y vivenciar alegría, gozo, satisfacción y placer, cuando ese alguien o algo que brinda la posibilidad de disfrute se pierde o bien no satisface las condiciones antes mencionadas, se puede presentar una reacción básica de Retiro y alejamiento de la situación que produce una pérdida o dolor; a nivel paradojal se produce una reacción asociada al Activismo o esfuerzo por tratar de cubrir el desagrado con el desarrollo de una o varias actividades; por otra parte puede presentarse como una reacción de tipo agresiva, la presencia de Rabia, diferenciándose de la manifestación de odio expresada en la primera motivación, en vista de que no busca destruir si no que busca despertar al otro perdido, que ya no está al alcance de sí mismo; finalmente como reacción vinculada al reflejo de muerte, se puede apreciar la presencia de la Resignación o agotamiento, cuando todo ha fallado y pueden emerger trastornos del ánimo tales como la 
Depresión (Längle, 2000).

Como tercera motivación de la existencia se encuentra asociada a la mismidad y su diferenciación con la otroredad, la capacidad de reconocer las propias características, identificarse como una persona con cualidades individuales, surgiendo el siguiente planteamiento "Yo soy yo, ¿pero me permito ser como yo soy?", surgiendo la necesidad de esclarecer las posibilidades de que el solo hecho de ser una persona determinada es permitido por alguien más o bien debe surgir una adaptación a como los demás quieren que uno sea (Längle, 2000). Como condiciones para satisfacer la presente motivación se encuentra la necesidad de consideración de otro, de ser respetado en mi propia identidad y como persona en mis necesidades, también se presenta la necesidad de recibir un trato justo siendo recompensado por el hecho solo hecho de presentarme ante mis figuras significativas como yo, finalmente una tercera condición se asocia a la posibilidad de recibir aprecio por parte de otros y de mí mismo, el surgimiento de una instancia de valoración personal a nivel interpersonal e individual (Längle, 2003).

Ante la no satisfacción de las condiciones necesarias anteriormente mencionadas, surgen reacciones a nivel básico asociadas a Tomar distancia, el cual se evidencia en la tendencia al alejamiento de otro, no estableciéndose una vinculación duradera; por otra parte surge como reacción paradojal el Sobreactuar o dar la razón, asociado esto al desarrollo de una sobreactuación para evidenciar que una situación asociada al dolor no es molesta, aparentando un sentimiento de agrado a modo de que el otro no tengo control sobre el individuo, el acto de dar la razón se asocia a entregar la razón a la figura del agresor como un acto de defensa no de convicción; en relación una reacción de tipo agresiva se presenta la Ira o fastidio, encontrándose la ira dirigida a otro, ubicando una barrera que no pueda generar daño o sufrimiento, por otra parte el fastidio se asocia a un castigo infringido a sí mismo, por no haber previsto lo que me hiere actualmente con antelación; finalmente como reflejo de muerte se encuentra la Disociación (Splitting), visualizada como una reacción de carácter histérico, se aprecia la separación entre la cognición y la emoción, no existiendo un espacio de conexión entre el individuo y su sentir, produciéndose un alejamiento absoluta ante la experiencia que pueda resultar dolorosa (Längle y Traverso, 2013).

De las reacciones de coping anteriormente mencionadas, surgen los trastornos de personalidad asociados al sí mismo. La combinación de los efectos psicopatológicos de la segunda y tercera motivación, entregan directrices respecto a la vivencia existencial y el sufrimiento histórico de una persona que no ha contado con los cuidados y protección, como tampoco con la consideración de sus figuras a nivel vincular, siendo el alejamiento y la separación de la sensación, una imposibilidad básica que dificulta el vínculo a nivel corporal con el acto de vivir la vida.

Finalmente y como ultima motivación fundamental se encuentra asociada al existir y la motivación o sentido asociado a dicho acto, estableciendo el siguiente 
planteamiento "Yo estoy aquí, ¿pero para que es bueno que yo esté aquí", encontrándose como condiciones asociadas a esta motivación, la existencia de un campo de acción donde sentirse requerido, necesitado, exigido, visualizando la posibilidad de ser productivo, también se encuentra la vinculación con un contexto mayor, la cual se asocia a un horizonte que brinda productividad y actividad, pudiendo este asociarse a la familia, una empresa, un ideal, etc; finalmente el valor en el futuro se vincula a que las acciones desarrolladas deben generar frutos y trascendencia como acción enfocada en brindar un sentido a la propia existencia (Längle, 2000).

Como reacciones posibles, se encuentra la presencia de una reacción básica asociada al establecimiento de Relaciones provisorias, no existiendo en este tipo de reacción el desarrollo de compromisos o vinculaciones profundas, asociándose esto a una existencia superficial; por otra parte se evidencia una reacción de tipo paradojal asociada al Fanatismo e idealización, comprendiendo el fanatismo como una reacción ante la amenaza de vivir una vida con una falta de sentido, desarrollo una actividad de manera empeñosa y obsesiva, a modo de no dar lugar a una duda la cual puede hacer visible la carencia de sentido, por otra parte la idealización es vivenciada como una idea o persona a la cual se le brinda una importancia sobrevalorada, a modo de reemplazar una falta de sentido; como tercera reacción asociada a la agresividad, se encuentra el Cinismo y Sarcasmo, siendo evidenciado a través de la destrucción y agresión de otros a través del vandalismo, a modo de evidenciar una falta de sentido y carencias a nivel personal, siendo el cinismo y sarcasmo también manifestaciones de esto; finalmente se presenta como reflejo de muerte una reacción de Desesperación, la cual se asocia al establecimiento de una posición de victimización y pasividad, además de un fuerte sentido de desesperanza donde no hay espacio para la acción (Längle, 2000).

El desarrollo del análisis existencial brinda una estructura novedosa que permite la generación de una psicoterapia fenomenológica, cuyo acercamiento mantiene una vinculación a la existencia desde una mirada desprejuiciada, pero además abierta a la posibilidad del establecimiento de una vinculación desde la interacción dialógica, siendo posible el desarrollo de interpretaciones vivenciales, basadas principalmente en brindar un fuerte énfasis en el desarrollo histórico individual, permitiendo que el individuo sea capaz de tomar decisiones de manera particular, orientado al respeto de la propia autonomía en el acercamiento a las problemáticas que lo vinculan a un proceso de apoyo psicoterapéutico. Si bien el análisis existencial cuenta con una amplia base teórica que puede conllevar la posibilidad de una imposición teórica por sobre el valor de la vivencia personal en la ejecución por parte de terapeutas inexpertos, es importante considerar que el desarrollo de un acercamiento e intencionalidad fenomenológicas representan importantes novedades en este nuevo modelo terapéutico, existiendo desde esta perspectiva la posibilidad de un ejercicio de suspensión teórica, asociada al ideal del discurso como posibilidad de interpretación y comprensión de la experiencia 
personal.

\section{La Renovación de la Mirada Posracionalista, un Acercamiento a la Fenomenología Hermenéutica}

El surgimiento del modelo Posracionalista en terapia, emerge como una alternativa cognitivista, asociada a la comprensión y abordaje del desarrollo de la identidad a nivel personal, respondiendo de la mano de Vittorio Guidano a un desarrollo basado en la articulación de una psicoterapia basada en la autoobservación, como ejercicio de reconstrucción de significados a nivel personal. La pronta partida de Guidano, conlleva cambios sustanciales en cuanto al desarrollo de una visión epistemológica continental por parte de Giampiero Arciero, quien incorpora la mirada fenomenológica hermenéutica, como elementos centrales de la actualización del presente modelo.

Guidano (1987), propone una crítica fundamentada al desarrollo del conocimiento objetivo de todas las cosas, como explicación en base a criterios de verdad, es desde esta perspectiva que se establece una clara observación asociada a que el desarrollo de una experiencia individual no se basa solo en fenómenos perceptivos, sino de la experimentación de los propios límites de la persona, los cuales se encuentran asociados a determinaciones afectivas y lingüísticas que convergen en el reordenamiento de un yo que genera significados particulares (Adasme, 2018). Es desde esta perspectiva que surge una renovación de la percepción de la construcción de la realidad por parte del individuo, la cual se asocia a la esfera de la adaptación desde las posibilidades emergentes en la emocionalidad y el lenguaje particular, de acuerdo a lo establecido por Guidano (1994), se puede comprender con mayor claridad este asunto: "El conocimiento va desde lo interno a lo externo, pertenece en primera instancia al organismo, parte de exigencias propias, muchas de ellas genéticamente determinadas. El conocimiento es la manera en la cual el organismo transforma el ambiente para encontrar su adaptación" (citado en Adasme, 2016, p. 6).

Es en su vinculación a las ideas de Maturana y Varela (2009), que Guidano desarrolla una visión Posracionalista, basada en los conceptos de sistemas cerrados y autopoiesis, como también en las ideas propuestas por Morin (1977), entre otros (Moltedo, 2008). Si bien en un comienzo se apega a conceptos asociados al desarrollo del self, se visualiza un alejamiento de dichos postulados, al adoptar una visión más cercana a la idea de interpretación y comprensión desde una visión de narrativa-hermenéutica de Paul Ricoeur (Moltedo, 2008). Ya asociado a una visión constructivista del conocimiento humano, se plantea la imposibilidad del racionalismo en la comprensión profunda del ser humano, siendo el ser humano un constructor activo de sus propios significados y realidades y posteriormente integrados como información por medio de axiomas lógicos (Balbi, 1994).

En consideración de la importancia a la afectividad entregada por Guidano en el desarrollo del modelo Posracionalista, recurre a los planteamientos de John 
Bowlby (1988), respecto a su teoría del apego y como el desarrollo psicoafectivo que surge a partir de la vinculación en etapas tempranas, contribuye a la formación de la identidad en el individuo (León y Tamayo, 2011). En la cuestión asociada al desarrollo de la identidad personal, siendo este un tema central en los planteamientos de Vittorio Guidano, siendo el nivel de la experiencia inmediata, lo que permite al individuo percibir más de lo que experimente o bien percibe conscientemente (Guidano, 1987). Desde el lenguaje, es posible establecer un ordenamiento experiencial organizado, denominado estructura narrativa de la experiencia humana, estableciendo que toda experiencia humana presenta un orden cronológico, temático y causal (Guidano, 1994).

Es propicio establecer una diferenciación en cuanto al desarrollo de la experiencia inmediata la cual es de carácter pre-reflexivo, a diferencia de la historia personal vivida e integrada, la cual puede ser reflexionada, organizada y distinguida con claridad, permitiendo además en la experiencia inmediata ya transcurrida, desarrollar un proceso reflexivo y de reconfiguración (Arciero, 2009). Guidano (1987), desarrolla un modelo de organización de significados personales (OSP), las cuales pueden definirse como: "La organización de conocimiento personal que emerge gradualmente en el curso del desarrollo individual. Cada individuo, aunque vive en una realidad socialmente objetiva y compartida, construye activamente en un nivel superior de experiencia su visión propia y única desde su interior" (p. 92).

Ante la prematura partida de Vittorio Guidano en 1999, quien presentaba el ideal de continuar desarrollando el presente modelo, Giampiero Arciero actual presidente del IPRA(Istituto di Psicologia e Psicoterapia Cognitiva Post-Razionalista, Roma, Italia), se ha encargado de desarrollar un nuevo marco de intervención, considerando en esta renovación, una tradición filosófica continental que postula una máxima integración a un ideal asociado a la inmersión en la experiencia particular desde la teoría de Edmund Husserl, Martin Heidegger y Paul Ricoeur, surge el Posracionalismo Fenomenológico Hermenéutico.

Dentro de las bases asociadas a estos nuevos planteamientos, se encuentra la consideración por parte de Arciero quien establece que los postulados establecidos por Guidano se apoyan en las ciencias naturales, planteando que la comprensión de un sistema complejo como el ser humano representa un problema desde dicha visión, más aún el abordaje de la subjetividad desde una corriente causal-lineal (Arciero, 2009). De acuerdo a Arciero, la posibilidad de acceso a la subjetividad individual, comprendida como consciencia con intencionalidad se asocia a las posibilidades entregadas por la reducción fenomenológica, influenciado claramente por Husserl, poniendo entre paréntesis la experiencia de la figura observadora, a modo de obtener un alumbramiento de la experiencia particular de la experiencia vivida por el individuo (Arciero, 2009).

En la búsqueda de una definición del sí mismo, Arciero adopta desde Ricoeur, el concepto de Mismidad, pudiendo esta ser definida como la dimensión estructural 
del ser que con independencia del tiempo transcurre con un significado permanente, por otro lado el concepto de Ipseidad, se asocia a la definición identitaria o del sí mismo a partir del encuentro situacional a nivel histórico, estableciendo una definición de la propia existencia en base a la interacción con el entorno inmediato (Arciero, 2009). De acuerdo al sentido de la Ipseidad, es justo referir que la experiencia de ser uno mismo es discontinua, siendo la continuidad de relacionarse con el mundo de maneras diferentes, la que brinda un verdadero sentido de continuidad (Arciero, 2008).

En cuanto a la emergencia de la Hermenéutica, se desprende en la comprensión de la facticidad del ser en el mundo, comprendiendo al ser humano como un ser hermenéutico en el sentido de que experimentar, es posicionarse en relación a la alteridad respecto de un modo particular de sentirse, de interpretarse, es decir de ser en cada situación histórica (Adasme, 2016). Para Arciero, la narrativa personal siempre se asocia a una corporalidad en constante modificación, sujeto a situaciones, experiencias y posibilidades, estando este cuerpo sujeto a estímulos sensorioperceptivos y emocionales de un ser situado en respuesta en la interacción con otros y con el mundo (Adasme, Ferrer y Zagmutt, 2013).

El desarrollo de una construcción lingüística de significados individuales, los cuales serán compartidos a nivel interaccional, se encuentra sujeto a relaciones emocionales como respuesta a las reacciones emocionales de los otros (origen de las emociones sociales) y también de las respuestas emocionales básicas asociadas a dicho marco relacional, estas emociones brindan propiedad a la experiencia vivenciada a nivel particular (Arciero y Bondolfi, 2009). Un individuo es capaz de conocerse al establecer la posibilidad de realizar una auto interpretación, la cual es dada a partir de los relatos históricos como también los de ficción realizados por el individuo, es decir conjugando los relatos facticos y los fabulados es posible establecer una historia personal, transmitida como historia vivida (Arciero, 2008).

A nivel psicoterapéutico, se estima que mediante el desarrollo del dialogo entre terapeuta y paciente, se puede favorecer que el otro llegue a una comprensión de sí mismo como sujeto histórico con significados (Arciero, 2012). El autor plantea que el desarrollo terapéutico se centra en la apropiación y reconfiguración de la experiencia en una renovada narración a nivel individual, estableciendo el desafío por parte del terapeuta de lograr un ejercicio de abstracción de significados a partir del relato, respecto a la vivencia emocionalmente vivenciada, más allá de una mirada apriorística desde categorías previamente definidas, siendo relevante la consideración de la dimensión experiencial a partir de la interpretación de las vivencias de la vida fáctica.

Arciero (2014), establece una propuesta metodológica, orientada al desarrollo de momentos terapéuticos centrados en el desarrollo de una psicoterapia posracionalista fenomenológico hermenéutica. Los momentos son los siguientes:

1. Acceso a la historia, interpretando el significado situado del presente y en pugna con el horizonte futuro de posibilidades. Posibilitando la identifi- 
cación de relatos que permitan la identificación de la pérdida del sentido de intimidad consigo mismo por parte del consultante, propiciando el reconocimiento de los posibles fenómenos que han generado una ruptura a nivel individual.

2. Deconstrucción del relato que encubre y mantiene el síntoma, correspondiendo a un proceso guiado centrado en la identificación de:

A) Como se tiene la experiencia vivida

B) Como se está relacionado a ella

C) Como se narra el sujeto a si mismo

Mediante el presente ejercicio se espera conectar el significado de cierta experiencia a las situaciones respectivas que le dan sentido.

3. Comprensión/Actualización asociada a la posibilidad de lograr una apropiación por parte del individuo de los significados y experiencias vivenciadas, a modo de desarrollar una renovación basada en la reconstrucción de nuevos posibles significados que propicien la movilización del paciente.

4. Construcción asociada a la renovación de la Ipseidad, la cual brinda nuevas trayectorias de sentido a nivel particular, propiciando la generación de un acercamiento a las múltiples posibilidades del sí mismo en el acto del vivir.

5. La renovación de la Ipseidad se asocia a un nuevo acceso a las experiencias vividas, respondiendo a una nueva forma de narrarse y sentirse en la vinculación entre el individuo y su entorno.

Es factible referir que la renovación de la terapia posracionalista se vincula a una necesidad de vincular el ejercicio terapéutico a posibilidades que brinden una mayor apertura al mundo de las vivencias particulares, desarrollando una comprensión ontológica que beneficia el ejercicio de construcción de futuras psicoterapias apegadas al desarrollo de intervenciones de carácter apriorístico. Se puede visualizar en la presente renovación, una practicidad en el objetivo principal planteado en la presente investigación, contando con una articulación que permite poner en práctica a nivel terapéutico, los postulados fenomenológicos hermenéuticos asociados al abordaje psicopatológico, sin embargo resta visualizar los posibles beneficios asociados al desarrollo de esta práctica, la cual si bien establece un respeto a la esfera de significados particulares de la experiencia vivida, aún se encuentra en continuo desarrollo, lo cual conlleva un estudio sostenido en cuanto a su aplicación con pacientes que presentan trastorno de personalidad severos.

\section{Discusión}

A partir de los postulados revisados en el presente trabajo, se han dilucidado ciertas dudas relativas a la aplicación de los postulados de la fenomenología a nivel clínico, pudiendo afirmar que el desarrollo de modelos psicoterapéuticos que favorezcan una vinculación a la experiencia fáctica, representa una apasionante 
posibilidad en cuanto al alcance de la experiencia vivida como foco central de la intervención. Desde Karl Jaspers a Giampiero Arciero, se ha establecido la importancia de cómo desde una práctica clínica basada en el desprejuicio con ausencia de un determinismo teórico, se obtiene un acceso a la subjetividad en la práctica clínica, aspecto regularmente desechado en los modelos tradicionales. Para una revisión integrada de los planteamientos expuestos en el presente trabajo, se recomienda revisar el cuadro ubicado en el apartado Anexo 1.

A partir de una revisión de la actualidad a nivel psicoterapéutico, es innegable destacar el importante legado que estos exponentes han entregado al ejercicio clínico, fundado en un apoyo a partir de la inmersión en la experiencia, como puerta de ingreso a dimensiones inaccesibles desde contenidos meramente explicativos, surgiendo la necesidad de establecer una rearticulación que comprometa una significativa renovación paradigmática, basada en un acercamiento hacía el ejercicio de la comprensión por sobre la explicación causal.

Es factible referir que la evolución y adaptación de modelos de intervención que respondan a una línea fenomenológica, se asocia a un arduo trabajo por parte de la figura del terapeuta, siendo necesario realizar un análisis detallado respecto al desarrollo inicial de una epoche a nivel terapéutico, instancia que conlleva un trabajo de definición ontológica profunda, la cual permita la mantención de un interés genuino centrado en un acercamiento desprejuiciado como una alternativa efectiva durante el desarrollo del ejercicio terapéutico.

\section{Referencias Bibliográficas}

Adasme, D. (2018). Actualización del Posracionalismo. Recuperado de: http://www.revistaposracionalismo.cl/ 2018/04/08/actualizacion-del-posracionalismo/

Adasme, D. Ferrer, M. y Zagmutt, A. (2013). Actualizaciones en Psicología y Psicoterapia Posracionalista. Seminario realizado por la Universidad del Salvador, Buenos Aires, Argentina.

Arciero, G. (2008). Tras las huellas del si mismo. Buenos Aires, Argentina: Amorrortu.

Arciero, G. (2009). Sul post-razionalismo a margine di un confronto fra Cutolo e Mancini. Delta, 1-8.

Arciero, G. (2012, noviembre). Apuntes Seminario de Personalidad tendiente a Trastornos Depresivos y Método Clínico. Seminario organizado por Universidad Adolfo Ibáñez y la Sociedad de Terapia Cognitiva Posracionalista. Santiago de Chile, Chile.

Arciero, G. (2014). Apuntes Seminario Articulación metódica de la psicoterapia fenomenológica. Universidad de Santiago de Chile, Chile.

Arciero, G. y Bondolfi, G. (2009). Selfhood, Identity and Personality Styles. Oxford, Reino Unido: Willey Blackwell.

Balbi, J. (1994). Terapia cognitiva posracionalista. Conversaciones con Vittorio Guidano. Buenos Aires, Argentina: Biblos.

Bazzi, T. y Fizzotti, E. (1989). Guía de logoterapia. Humanización de la psicoterapia. Barcelona, España: Herder.

Blankenburg, W. (1984). Unausgeschöpftes in der Psychopathologie von Karl Jaspers. Der Nervenart, 55 (9), 447 460 .

Bowlby, J. (1988). A secure base: Parent-child attachment and healthy human development. Nueva York, NY: Basic Books.

Chaslin, Ph. (1912). Éléments de sémiologie et clinique mentales. Paris, Francia: Asselin et Houzeau.

Fizzotti, E. (1977). De Freud a Frankl. Interrogantes sobre el vacio existencial. Pamplona, España: EUNSA. 
Frankl, V. (1926). Zur Psychologie des Intellektualismus. Internationale Zeitschrift für Individualpsychologie, 4, 326-332.

Frankl, V. (1986). Ante El vacío Existencial. Hacia una humanización de la psicoterapia. Barcelona, España: Herder.

Frankl, V. (1988). El hombre en busca de sentido. Barcelona, España: Herder.

Frankl, V. (1994). El hombre doliente. Barcelona, España: Herder.

Frankl, V. (2 $2^{\mathrm{a}} \mathrm{ed}$.) (2003). Lo que no está escrito en mis libros. Memorias. Buenos Aires, Argentina: San Pablo.

Frankl, V. (2007). Logoterapia y análisis existencial. Barcelona, España: Herder.

Guidano, V. (1987). Complexity of the self. Nueva York, NY: Guilford Press.

Guidano, V. (1994). El sí mismo en proceso: hacia una terapia cognitiva posracionalista. Barcelona, España: Paidós.

Heidegger, M. (1997). Ser y Tiempo. Santiago de Chile, Chile: Editorial Universitaria.

Hersch, J. (2002). Karl Jaspers. Lausana, Suiza: L’Age d'Homme.

Husserl, E. (1962). La filosofia como ciencia estricta. Buenos Aires, Argentina: Nova.

Jaspers, K. (1912). Die phänomenologische Forschungsrichtung in der Psychopathologie. Z gesam Neurol Psychiat, 9, 391-408.

Jaspers, K. (1950). Die moderne Wissenschaft. Physikalische Blätter, 6(11), 481-488.

Jaspers, K. (1951). Rechenschaft und Ausblick. Reden und Aufsatze. München, Alemania: Piper.

Jaspers, K. (1959). Allgemeine Psychopathologie. Berlin, Alemania: Springer.

Jaspers, K. (1965). Wahrheit und Leben. Ausgewählte Schriften. Zurich, Suiza: Buchklub ex libris.

Längle, A. (1990). Existential analysis psychotherapy. International Forum for Logotherapy, 13(1), 17-19.

Längle, A. (2000, mayo). Las motivaciones fundamentales de la existencia. Seminario Buenos Aires, Argentina.

Längle, A. (2003). The Search for Meaning in Life and the Fundamental Existential Motivations. Psychotherapy in Australia, 10(1), 22-27.

Längle, S. y Traverso, G. (2013). Vivir la propia vida. Análisis Existencial desde la praxis. Santiago de Chile, Chile: Mandagora Ediciones.

León, A. y Tamayo, D. (2011). La psicoterapia cognitiva posracionalista: Un modelo de intervención centrado en la construcción de la identidad. Katharsis 12, 37-58.

Maturana, H. y Valera, F. (2009). El árbol del conocimiento. Las bases biológicas del conocimiento humano. Santiago de Chile, Chile: Editorial Universitaria.

Martinez, E. (2012). El dialogo socrático en la psicoterapia. Bogota, Colombia: SAPS.

Moltedo, A. (2008). La evolución de la obra y el modelo de Vittorio Guidano: Notas histórico biográficas. Revista de Psicología, 17(1), 65-85.

Mook, B. (2010). Hermeneutic Phenomenology and Psychotherapy. Les Collectifs du Cirp, 1 (édition spéciale), 209-222.

Morin, E. (1977). La Méthode 1: La Nature de la Nature. Paris, Francia: Seuil.

Parnas, J., Sass, L. A. y Zahavi, D. (2013). Rediscovering psychopathology: The epistemology and phenomenology of the psychiatric object. Schizophrenia Bulletin, 39(2), 270-277.

Salomón, A. y Díaz del Castillo, J. (2015). Encontrando y realizando sentido. Diálogo socrático y ejercicios vivenciales en logoterapia. Lima, Perú: Unifé.

Scheler, M. (1957). El puesto del hombre en el cosmos. Buenos Aires, Argentina: Losada. 


\section{Anexo 1}

Tabla 1. Cuadro resumen.

\begin{tabular}{|c|c|c|c|c|}
\hline Autores & Jaspers & Frankl & Längle & Arciero \\
\hline Conceptos & $\begin{array}{l}\text { "Método } \\
\text { biográfico" } \\
\text { orientación a la } \\
\text { identificación de } \\
\text { la "vivencia" } \\
\text { asociada a los } \\
\text { trastornos } \\
\text { mentales. } \\
\text { Fenomenología } \\
\text { en la práctica } \\
\text { clínica. }\end{array}$ & $\begin{array}{l}\text { "Análisis } \\
\text { Existencial" } \\
\text { "Logoterapía" la } \\
\text { búsqueda del } \\
\text { sentido como } \\
\text { práctica } \\
\text { terapéutica } \\
\text { asociada a la } \\
\text { superación del } \\
\text { vacío existencial. }\end{array}$ & $\begin{array}{l}\text { "Análisis } \\
\text { Existencial" } \\
\text { "Motivaciones } \\
\text { fundamentales } \\
\text { de la existencia" } \\
\text { el desarrollo de } \\
\text { un modelo } \\
\text { fenomenológico } \\
\text { que permite la } \\
\text { identificación de } \\
\text { reacciones } \\
\text { psicodinámicas y } \\
\text { su respectiva } \\
\text { resolución, a } \\
\text { partir del dialogo. }\end{array}$ & $\begin{array}{l}\text { "Posracionalismo } \\
\text { Fenomenológico } \\
\text { Hermenéutico" } \\
\text { renovación del } \\
\text { posracionalismo } \\
\text { de Guidano, } \\
\text { incorporando los } \\
\text { conceptos de } \\
\text { Ipseidad, } \\
\text { historia } \\
\text { encarnada. Se } \\
\text { adhiere a los } \\
\text { postulados de } \\
\text { Heidegger, } \\
\text { Husserl y } \\
\text { Ricoeur. }\end{array}$ \\
\hline Propósito & $\begin{array}{l}\text { Comprender la } \\
\text { experiencia de } \\
\text { cada paciente en } \\
\text { relación a una } \\
\text { patología } \\
\text { determinada. }\end{array}$ & $\begin{array}{l}\text { Favorecer la } \\
\text { búsqueda de un } \\
\text { sentido } \\
\text { particular, a } \\
\text { modo de superar } \\
\text { la emergencia de } \\
\text { vacíos } \\
\text { existenciales y } \\
\text { posibles } \\
\text { neurosis. }\end{array}$ & $\begin{array}{l}\text { Favorecer la } \\
\text { adaptación por } \\
\text { parte del } \\
\text { individuo, } \\
\text { desarrollando la } \\
\text { toma de } \\
\text { decisiones libres } \\
\text { que permitan a la } \\
\text { persona lidiar } \\
\text { con la vida y el } \\
\text { mundo. }\end{array}$ & $\begin{array}{l}\text { Establecer una } \\
\text { psicoterapia } \\
\text { orientada a la } \\
\text { renovación del } \\
\text { sentido de sí } \\
\text { mismo y de la } \\
\text { esfera de } \\
\text { significados } \\
\text { históricos, a } \\
\text { modo de que el } \\
\text { individuo pueda } \\
\text { hacerse cargo } \\
\text { de sí mismo, } \\
\text { estableciendo } \\
\text { una nueva } \\
\text { posición }\end{array}$ \\
\hline Tarea & $\begin{array}{l}\text { Desarrollar un } \\
\text { método que } \\
\text { permita realizar } \\
\text { un seguimiento } \\
\text { histórico y } \\
\text { vivencial de la } \\
\text { experiencia } \\
\text { individual }\end{array}$ & $\begin{array}{l}\text { Mediante la } \\
\text { Logoterapia } \\
\text { favorecer a la } \\
\text { búsqueda de } \\
\text { sentido a nivel } \\
\text { individual, como } \\
\text { fuerza } \\
\text { motivadora de la } \\
\text { existencia. }\end{array}$ & $\begin{array}{l}\text { Reconocer las } \\
\text { condiciones para } \\
\text { tener una vida } \\
\text { plena. }\end{array}$ & $\begin{array}{l}\text { Dar cuenta del } \\
\text { problema de la } \\
\text { intimidad, } \\
\text { unidad y } \\
\text { continuidad del } \\
\text { sí mismo, } \\
\text { estableciendo la } \\
\text { pregunta por el } \\
\text { "quien vivencia" }\end{array}$ \\
\hline
\end{tabular}

